\title{
SCIENTIFIC REPORTS

\section{OPEN Prenatal and birth predictors of objectively measured physical activity and sedentary time in three population-based birth cohorts in Brazil}

\author{
Ding Ding $\mathbb{1}^{1 *}$, Gregore I. Mielke ${ }^{2,3}$, Inacio Crochemore M. Silva $\mathbb{D}^{4}$, Fernando C. Wehrmeister $\mathbb{C}^{4}$, \\ Bernardo L. Horta $\mathbb{1}^{4}$, Soren Brage ${ }^{5}$, Pedro C. Hallal ${ }^{2}$ \& Ulf Ekelund ${ }^{6}$ \\ Physical inactivity is a global pandemic with no signs of improvement. Prolonged sitting time is an \\ emerging risk factor that exacerbates the health consequences of physical inactivity. Both behaviours \\ are influenced by various individual and environmental factors but it remains unknown whether \\ early-life exposures "program" these behaviours in later life. The current evidence is limited by a small \\ number of studies which were primarily conducted in high-income countries, and a narrow range of \\ early-life variables examined. Using data from three population-based Brazilian birth cohorts (analytical \\ samples: $\mathbf{n}=\mathbf{2 7 4 0}$ for 1982 cohort, aged 30 years; $\mathrm{n}=3592$ for 1993 cohort, aged 18; $\mathrm{n}=2603$ for \\ 2004 cohort, aged 6), we show that being female and higher family socioeconomic status at birth are \\ strong and consistent predictors of lower physical activity and higher sedentary time from childhood \\ to adulthood. Meanwhile, higher birth weight and lower birth order may also predict lower physical \\ activity and higher sedentary time. Our findings are distinct from evidence from high-income countries, \\ suggesting the importance of broader socioeconomic context in determining individual's activity \\ patterns through the life- course. Such evidence is essential for understanding the biological etiology \\ and socioeconomic context of physical activity and sedentary behaviour at an early stage in life.
}

Physical inactivity is responsible for chronic diseases, premature mortality ${ }^{1}$ and substantial economic burdens ${ }^{2}$. With one in three adults and four in five adolescents worldwide failing to achieve the recommended levels of physical activity ${ }^{3}$ and no signs of improvement at the population level ${ }^{4}$, the pandemic of physical inactivity is one of the biggest challenges to global health. Sedentary behaviour, on the other hand, is a distinct class of waking behavior in a sitting, reclining, or lying posture with an energy expenditure $\leq 1.5$ Metabolic Equivalents ${ }^{5}$. Increasing evidence suggests that prolonged sedentary time may elevate the risk of chronic diseases and mortality in adults and the combination of prolonged sitting time and low levels of physical activity may be particularly detrimental to health ${ }^{6}$.

Understanding the causes of physical inactivity and prolonged sedentary time is essential for developing interventions to improve public health. Hence, many studies have been conducted to understand why some people are more active than others. It is generally understood that physical activity and sedentary behaviour are jointly influenced by a range of individual, interpersonal, environmental and policy factors across different stages in life ${ }^{7,8}$. However, one consistently understudied area is whether and how prenatal, birth and early life factors influence

\footnotetext{
${ }^{1}$ Prevention Research Collaboration, School of Public Health, and Charles Perkins Centre, University of Sydney, Camperdown, NSW, 2006, Australia. ${ }^{2}$ Postgraduate Program in Epidemiology, Federal University of Pelotas, Brazil Rua Marechal Deodoro, 1160 - $3^{\circ}$ Piso, Bairro Centro, Pelotas, RS, Cep: 96020-220, Caixa Postal 464, Brazil. ${ }^{3}$ School of Human Movement and Nutrition Sciences, University of Queensland, St Lucia, QLD, 4067, Australia. ${ }^{4}$ Postgraduate Program in Epidemiology and International Center for Equity in Health, Federal University of Pelotas, Rua Marechal Deodoro, 1160 - $3^{\circ}$ Piso, Bairro Centro, Pelotas, RS, Cep: 96020-220, Caixa Postal 464, Brazil. ${ }^{5}$ MRC Epidemiology Unit, University of Cambridge School of Clinical Medicine, Cambridge, CB20SL, UK. ${ }^{6}$ Norwegian School of Sports Sciences, PO Box 4014, Ulleval Stadion 0806, Oslo, Norway. *email: melody.ding@sydney.edu.au
} 
these behaviours ${ }^{9,10}$. The Developmental Origins of Health and Disease Theory, based on Barker's hypothesis ${ }^{11}$, emphasises the important roles of non-optimal growth and early life environments in "programming" health and disease outcomes in utero and during early life ${ }^{12}$. While most studies focused on early "programming" of physiological outcomes, such as adiposity and hypertension ${ }^{13}$, few explored behavioral outcomes ${ }^{14}$. Given that physical activity and sedentary behaviour are habitual in nature and can track from childhood to adulthood ${ }^{15,16}$, it is logical to hypothesize that some of their determinants may manifest early in life, potentially before or at birth ${ }^{9}$. Indeed, animal studies suggest a biologically plausible programming effect of physical activity and sedentary behaviour ${ }^{17,18}$. These studies suggest that an unfavourable, under- or over-nourished prenatal environment appears to program lower levels of spontaneous physical activity, persistent into adulthood ${ }^{18}$.

To date, a small number of epidemiological studies have examined the prenatal and birth predictors of physical activity and sedentary behaviour with the primary focus on birth weight and gestational age. Findings from these studies suggest that birth weight is likely to have a weak association with physical activity in childhood, adolescence $^{19}$, and adulthood ${ }^{20}$, but there is little evidence for an association of birth weight and gestational age with sedentary behaviour among young people ${ }^{9}$. A broader range of predictors, such as maternal weight status, birth order and type of delivery, have been less explored.

Furthermore, given that most studies on early life predictors of physical activity and sedentary behaviour to date were conducted in high-income countries ${ }^{9,19}$, and that childhood socioeconomic status plays an important role in later physical activity ${ }^{21}$, it is important to understand early life predictors of both biological and socioeconomic nature in a low- and middle- income country setting. Building such evidence is essential for understanding the biological etiology and socioeconomic context of physical activity and sedentary behaviour and for identifying and targeting those who may be at risk of an inactive lifestyle at an early stage in life.

To address these research gaps, we used data from three population-based birth cohorts of children and adults from Pelotas, Brazil, where rapid economic, lifestyle and epidemiological transitions were observed in the past few decades. We aimed to examine the association between a series of prenatal and birth predictors and objectively measured physical activity and sedentary behaviour later in life during childhood, adolescence and adulthood.

\section{Results}

This study is based on the 1982, 1993, and 2004 Pelotas Birth Cohorts. Pelotas is located in the Southern state of Rio Grande do Sul. These cohorts were comprised of live births, whose families lived in the urban area of Pelotas, delivered at maternity hospitals during the year of data collection. In 1982, 1993 and 2004, 6011, 5265, 4263 live births were reported in Pelotas, and 5914, 5249, 4231 were recruited to the cohort, representing $>98 \%$ of all live births in respective years in the city. Infants were measured, and their mothers interviewed within 24 hours of delivery. Prenatal and birth variables include those of both biological (i.e., sex, birth weight, gestational age, birth order, mode of delivery, and pre-pregnancy body mass index [BMI]) and socioeconomic nature (i.e., maternal education and family income). Cohort participants have been followed up at different times since birth. The present analyses are based on baseline and follow-up accelerometry data collected in 2010-2013 when participants were aged 30, 18, and 6 years, respectively. Three outcome variables were derived from accelerometry data: (1) measured physical activity of any intensity, which approximates the overall physical activity volume (milli-g), (2) moderate-to-vigorous intensity physical activity ([MVPA] $\mathrm{min} /$ day), which is conventionally considered activities within the "health enhancing" intensity threshold and a key component of physical activity guidelines, (3) sedentary time (min/day), which quantifies the total amount of sedentary behaviour.

The final analytical samples included 2740 participants from the 1982 cohort, 3592 from the 1993 cohort, and 2603 from the 2004 cohort, representing 46\%,68\%, and $62 \%$ of the baseline participants (Supplementary Fig. 1). As shown in Table 1, females accounted for slightly more than half of the 1982 and 1993 cohorts and less than half of the 2004 cohort. The distribution of birth weight was similar across cohorts with 7-9\% classified as low birthweight. Compared with the 1982 cohort, there was a higher proportion of preterm births and cesarean deliveries in the later cohorts. A lower proportion of mothers were classified as underweight and a higher proportion overweight or obese in the 2004 cohort compared with earlier cohorts. Across all cohorts, around 40\% of the participants were first-borns, and more than $40 \%$ were second- or third-born. The distribution of family income were similar across cohorts with the majority reporting a family income of $\leq 3$ times the minimum wage. The proportion of mothers with nine years of schooling or more was considerably higher in the 2004 cohort. The 2004 cohort had the highest levels of overall and moderate-to-vigorous physical activity and the lowest level of sedentary time (Table 1). Characteristics of the overall baseline samples and the analytical samples are comparable (Supplementary Table 1).

Across all three cohorts and outcomes, unadjusted and adjusted analyses yielded similar patterns of associations except for an attenuation of effect sizes in adjusted models in most cases. Results described below are primarily based on those from adjusted analyses.

With respect of overall physical activity, males and those with lower family income and maternal education at birth had higher overall physical activity (Table 2). Specifically, the average difference between male and female participants ranged between 5.1 and 9.2 milli-g across cohorts, with the difference between sex being the biggest in the youngest cohort, and the smallest in the oldest cohort. The association between maternal education levels and overall physical activity showed a dose-repose pattern with the average difference between the lowest and highest education categories being around 6.5 milli-g for the 2004 cohort, 5.3 milli-g for the 1993 cohort, and 3.1 milli-g for the 1982 cohort. The association between family income and overall physical activity was less dose-response, however, the lowest income category had consistently the highest levels of physical activity, which was around 2 milli-g higher than the highest income group across all three cohorts. Being born preterm was associated with slightly more overall physical activity (i.e., 2.0 mili-g) in the 1982 cohort only. Cesarean delivery was significantly associated lower overall physical activity in unadjusted models but was only statistically significantly 


\begin{tabular}{|c|c|c|c|}
\hline & 1982 Cohort & 1993 Cohort & 2004 Cohort \\
\hline & $(\mathrm{n}=2740)^{*}$ & $(\mathrm{n}=3592) *$ & $(n=2603)^{*}$ \\
\hline Age (years: mean[SD]) & $30.2(0.2)$ & $18.4(0.3)$ & $6.7(0.3)$ \\
\hline \multicolumn{4}{|l|}{ Characters at birth: $\mathrm{n}(\%)$} \\
\hline \multicolumn{4}{|c|}{ Family income (No. of minimum wages per month) } \\
\hline$\leq 1$ & $588(21.6)$ & $636(17.1)$ & $524(20.1)$ \\
\hline $1.1-3.0$ & $1383(50.7)$ & $1557(43.4)$ & $1204(46.3)$ \\
\hline $3.1-6.0$ & $505(18.5)$ & $866(24.1)$ & $599(23.0)$ \\
\hline $6.1-10.0$ & $141(5.2)$ & $286(8.0)$ & $154(5.9)$ \\
\hline$>10$ & $109(4.0)$ & $247(6.9)$ & $121(4.7)$ \\
\hline \multicolumn{4}{|l|}{ Maternal education (years of schooling) } \\
\hline 0 & $140(5.1)$ & $85(2.4)$ & $19(0.7)$ \\
\hline $1-4$ & $791(28.9)$ & $879(24.5)$ & $365(14.2)$ \\
\hline $5-8$ & $1215(44.4)$ & $1716(47.8)$ & $1092(42.4)$ \\
\hline $9+$ & $590(21.6)$ & $909(25.3)$ & $1100(42.7)$ \\
\hline \multicolumn{4}{|l|}{ Sex } \\
\hline Male & $1329(48.5)$ & $1764(49.1)$ & $1341(51.5)$ \\
\hline Female & $1411(51.5)$ & $1828(50.9)$ & $1262(48.5)$ \\
\hline \multicolumn{4}{|l|}{ Pre-pregnancy body mass index $\left(\mathrm{kg} / \mathrm{m}^{2}\right)$} \\
\hline$<18.5$ & $176(7.5)$ & $309(8.8)$ & $78(4.3)$ \\
\hline $18.5-24.9$ & $1625(69.5)$ & $2373(67.8)$ & $1071(59.2)$ \\
\hline $25-29.9$ & $421(18.0)$ & $645(18.4)$ & $446(24.7)$ \\
\hline$\geq 30$ & $116(5.0)$ & $172(4.9)$ & $213(11.8)$ \\
\hline \multicolumn{4}{|l|}{ Birth order } \\
\hline 1 & $1080(39.4)$ & $1426(39.7)$ & $1159(45.3)$ \\
\hline 2 or 3 & $1218(44.5)$ & $1635(45.6)$ & $1060(41.4)$ \\
\hline$\geq 4$ & $441(16.1)$ & $528(14.7)$ & $339(13.3)$ \\
\hline \multicolumn{4}{|l|}{ Gestational age (weeks) } \\
\hline$<37$ & $120(5.5)$ & $381(10.7)$ & $338(13.0)$ \\
\hline$\geq 37$ & $2075(94.5)$ & $3171(89.3)$ & $2263(87.0)$ \\
\hline \multicolumn{4}{|l|}{ Type of delivery } \\
\hline Vaginal & $2013(73.5)$ & $2474(68.9)$ & $1429(54.9)$ \\
\hline Cesarean & $727(26.5)$ & $1118(31.1)$ & $1174(45.1)$ \\
\hline \multicolumn{4}{|l|}{ Birth weight (g) } \\
\hline$<2500$ & $196(7.2)$ & $331(9.2)$ & $224(8.6)$ \\
\hline $2500-3499$ & $1711(62.4)$ & $2285(63.7)$ & $1663(63.9)$ \\
\hline$\geq 3500$ & $832(30.4)$ & $972(27.1)$ & $716(27.5)$ \\
\hline \multicolumn{4}{|l|}{ Accelerometer outcomes } \\
\hline Overall physical activity (milli-g) & $35.5(10.5)$ & $39.3(12.9)$ & $59.4(16.3)$ \\
\hline $\begin{array}{l}\text { Total moderate-to-vigorous physical activity } \\
\text { (minutes/day) }\end{array}$ & $25.8(31.4)$ & $43.0(42.2)$ & $44.6(34.7)$ \\
\hline Total sedentary time (minutes/day) & $679.6(88.8)$ & $688.5(90.5)$ & $607.1(74.0)$ \\
\hline
\end{tabular}

Table 1. Characteristics of the three birth cohorts in Pelotas, Brazil. *Numbers vary owing to missing values.

associated with physical activity in the adjusted model in the 1982 cohort; however, the average difference was only around 1.0 mili-g. Lower birth weight and higher birth order were associated with higher levels of physical activity in the 1993 and 2004 cohorts in the adjusted models. Specifically, the association between birthweight and overall physical activity was the strongest in the 1993 cohort where the average difference between the lowest and highest birthweight category was 3.8 mili-g. In terms of birth order, the difference between firstborns and those with the highest birth order (i.e., $4+$ ) was around 3 mili-g in both cohorts.

The overall pattern of associations regarding MVPA is similar to that regarding overall physical activity. Generally, males and those with lower family income and maternal education at birth had higher levels of MVPA. Specifically, the average difference in MVPA between sex was around 22 minutes per day in the 1993 and 2004 cohorts and around 13 minutes in the 1982 cohort. On average, those with the lowest maternal education (i.e., zero schooling) had more MVPA than those with the highest maternal education (i.e., 9+ years schooling) by a margin of 13 minutes per day in the 2004 cohort (not statistically significant), 17 minutes in the 1993 cohort and 9 minutes in the 1982 cohort. The association with income was similar, with the difference between the lowest and highest income categories being 7-10 minutes across cohorts. In the 1993 cohort, compared with participants whose mother was normal weight, those whose mother was underweight had around 6 more minutes of MVPA; those with a highest birth order (i.e., 4+) had nearly 8 more minutes of MVPA than firstborns; compared with 


\begin{tabular}{|c|c|c|c|c|c|c|c|c|c|c|c|c|}
\hline \multirow[b]{2}{*}{ Variables } & \multicolumn{4}{|c|}{1982 Cohort $(n=2740)^{c}$} & \multicolumn{4}{|c|}{1993 Cohort $(n=3592)^{c}$} & \multicolumn{4}{|c|}{2004 Cohort $(n=2603)^{c}$} \\
\hline & \begin{tabular}{|l|}
$\begin{array}{l}\text { Unadjusted } \beta \\
(95 \% \mathrm{CI})\end{array}$ \\
\end{tabular} & $\mathbf{p}$ & $\begin{array}{l}\text { Adjusted } \beta \\
(95 \% \text { CI })\end{array}$ & $\mathbf{p}$ & $\begin{array}{l}\text { Unadjusted } \beta \\
(95 \% \text { CI })\end{array}$ & $\mathbf{p}$ & $\begin{array}{l}\text { Adjusted } \beta \\
(95 \% \text { CI })\end{array}$ & $\mathbf{p}$ & \begin{tabular}{|l|} 
Unadjusted $\beta$ \\
$(95 \%$ CI $)$
\end{tabular} & $\mathbf{p}$ & $\begin{array}{l}\text { Adjusted } \beta \\
(95 \% \text { CI })\end{array}$ & p \\
\hline $\begin{array}{l}\text { Family income } \\
\text { (No of minimum } \\
\text { wages per month) }\end{array}$ & & $<0.001$ & & 0.027 & & $<0.001$ & & $<0.001$ & & $<0.001$ & & 0.009 \\
\hline$\leq 1$ & $5.33(3.20 ; 3.47)$ & & $\begin{array}{l}2.01(-0.29 ; \\
4.30)\end{array}$ & & $\begin{array}{l}\text { 6.02 (4.15; } \\
7.89)\end{array}$ & & $\begin{array}{l}2.25(0.32 \\
4.18)\end{array}$ & & $\begin{array}{l}4.89(1.69 ; \\
8.09)\end{array}$ & & $\begin{array}{l}2.36(-0.94 ; \\
5.67)\end{array}$ & \\
\hline $1.1-3.0$ & $4.54(2.48 ; 6.57)$ & & $\begin{array}{l}1.85(-0.29 ; \\
3.99)\end{array}$ & & $\begin{array}{l}5.18(3.47 ; \\
6.89)\end{array}$ & & $\begin{array}{l}2.25(0.51 ; \\
3.98)\end{array}$ & & $\begin{array}{l}2.94(-0.09 ; \\
5.97)\end{array}$ & & $\begin{array}{l}0.89(-2.21 ; \\
3.99)\end{array}$ & \\
\hline $3.1-6.0$ & $2.24(0.07 ; 4.41)$ & & $\begin{array}{l}0.59(-1.56 ; \\
2.74)\end{array}$ & & $\begin{array}{l}1.98(0.19 ; \\
3.79)\end{array}$ & & $\begin{array}{l}0.09(-1.67 ; \\
1.85)\end{array}$ & & $\begin{array}{l}0.48(-2.68 ; \\
3.64)\end{array}$ & & $\begin{array}{l}-0.63(-3.77 \\
2.50)\end{array}$ & \\
\hline $6.1-10.0$ & $1.95(-0.67 ; 4.56)$ & & $\begin{array}{l}1.03(-1.49 ; \\
3.56)\end{array}$ & & $\begin{array}{l}0.40(-1.18 ; \\
2.57)\end{array}$ & & $\begin{array}{l}-0.29 \\
(-2.32 ; 1.75)\end{array}$ & & $\begin{array}{l}-0.28(-4.14 \\
3.57)\end{array}$ & & $\begin{array}{l}-0.77(-4.52 \\
2.99)\end{array}$ & \\
\hline$>10$ & Ref & & Ref & & Ref & & Ref & & Ref & & Ref & \\
\hline $\begin{array}{l}\text { Maternal } \\
\text { education (year of } \\
\text { schooling) }\end{array}$ & & $<0.001$ & & $<0.001$ & & $<0.001$ & & $<0.001$ & & $<0.001$ & & 0.001 \\
\hline 0 & $4.00(2.08 ; 5.91)$ & & $\begin{array}{l}3.06(0.99 \\
5.14)\end{array}$ & & $\begin{array}{l}7.65 \text { (4.77; } \\
10.54)\end{array}$ & & $\begin{array}{l}5.31(2.51 ; \\
8.11)\end{array}$ & & $\begin{array}{l}8.12(0.78 ; \\
15.46)\end{array}$ & & $\begin{array}{l}6.53(-0.58 ; \\
13.65)\end{array}$ & \\
\hline $1-4$ & $4.60(3.49 ; 5.71)$ & & $\begin{array}{l}3.32(2.01 \\
4.63)\end{array}$ & & $\begin{array}{l}6.00(4.82 ; \\
7.17)\end{array}$ & & $\begin{array}{l}3.89(2.63 ; \\
5.16)\end{array}$ & & $\begin{array}{l}4.73(2.81 ; \\
6.64)\end{array}$ & & $\begin{array}{l}2.62(0.52 \\
4.73)\end{array}$ & \\
\hline $5-8$ & $3.74(2.72 ; 4.76)$ & & $\begin{array}{l}2.75(1.60 ; \\
3.89)\end{array}$ & & $\begin{array}{l}4.43(3.41 \\
5.45)\end{array}$ & & $\begin{array}{l}2.97 \text { (1.92; } \\
4.03)\end{array}$ & & $\begin{array}{l}3.50(2.15 ; \\
4.86)\end{array}$ & & $\begin{array}{l}2.30(0.86 \\
3.75)\end{array}$ & \\
\hline $9+$ & Ref & & Ref & & Ref & & Ref & & Ref & & Ref & \\
\hline Sex & & $<0.001$ & & $<0.001$ & & $<0.001$ & & $<0.001$ & & $<0.001$ & & $<0.001$ \\
\hline Male & Ref & & Ref & & Ref & & Ref & & Ref & & Ref & \\
\hline Female & $\begin{array}{l}-5.08(-5.85 \\
-4.31)\end{array}$ & & $\begin{array}{l}-5.14(-5.89 \\
-4.38)\end{array}$ & & $\begin{array}{l}-8.50(-9.30 \\
-7 ; 70)\end{array}$ & & $\begin{array}{l}-8.59 \\
(-9.37 ; \\
-7 ; 80) \\
\end{array}$ & & $\begin{array}{l}-9.06(10.26 \\
-7.86)\end{array}$ & & $\begin{array}{l}-9.19 \\
(-10.40 \\
-7.98) \\
\end{array}$ & \\
\hline $\begin{array}{l}\text { Pre-pregnancy } \\
\text { body mass index } \\
\left(\mathrm{kg} / \mathrm{m}^{2}\right)\end{array}$ & & 0.056 & & 0.039 & & 0.063 & & 0.245 & & 0.462 & & 0.523 \\
\hline$<18.5$ & $1.39(-0.26 ; 3.05)$ & & $\begin{array}{l}0.90(-0.66 ; \\
2.46)\end{array}$ & & $\begin{array}{l}2.25(0.73 ; \\
3.77)\end{array}$ & & $\begin{array}{l}1.01(-0.58 ; \\
2.27)\end{array}$ & & $\begin{array}{l}-3.77(-7.52 \\
-0.01)\end{array}$ & & $\begin{array}{l}-1.99(-5.29 \\
1.31)\end{array}$ & \\
\hline $18.5-24.9$ & Ref & & Ref & & Ref & & Ref & & Ref & & Ref & \\
\hline $25-29.9$ & $\begin{array}{l}-0.80(-1.94 ; \\
0.34)\end{array}$ & & $\begin{array}{l}-0.90(-2.00 ; \\
0.20)\end{array}$ & & $\begin{array}{l}0.07(-1.04 ; \\
1.19)\end{array}$ & & $\begin{array}{l}0.10(-0.94 ; \\
1.14)\end{array}$ & & $\begin{array}{l}-0.19(-2.00 \\
1.61)\end{array}$ & & $\begin{array}{l}-0.14(-1.77 \\
1.50)\end{array}$ & \\
\hline$\geq 30$ & $\begin{array}{l}-0.24(-2.24 ; \\
1.76)\end{array}$ & & $\begin{array}{l}-0.78(-2.69 ; \\
1.13)\end{array}$ & & $\begin{array}{l}-0.44(-2.43 \\
1.55)\end{array}$ & & $\begin{array}{l}-0.73 \\
(-2.58 ; 1.13)\end{array}$ & & $\begin{array}{l}0.03(-2.37 \\
2.43)\end{array}$ & & $\begin{array}{l}0.27(-2.05 ; \\
2.60)\end{array}$ & \\
\hline Birth order & & 0.020 & & 0.113 & & $<0.001$ & & $<0.001$ & & $<0.001$ & & 0.015 \\
\hline 1 & Ref & & Ref & & Ref & & Ref & & Ref & & Ref & \\
\hline 2 or 3 & $0.63(-0.23 ; 1.49)$ & & $\begin{array}{l}0.65(-0.19 ; \\
1.49)\end{array}$ & & $\begin{array}{l}1.41(0.50 \\
2.32)\end{array}$ & & $\begin{array}{l}1.10(0.25 ; \\
1.96)\end{array}$ & & $\begin{array}{l}0.86(-0.49 ; \\
2.21)\end{array}$ & & $\begin{array}{l}0.81(-0.54 ; \\
2.15)\end{array}$ & \\
\hline$\geq 4$ & $1.33(0.17 ; 2.50)$ & & $\begin{array}{l}0.81(-0.38 ; \\
2.01)\end{array}$ & & $\begin{array}{l}4.79(3.51 \\
6.06)\end{array}$ & & $\begin{array}{l}3.05(1.81 ; \\
4.29)\end{array}$ & & $\begin{array}{l}4.18(2.22 ; \\
6.14)\end{array}$ & & $\begin{array}{l}2.73(0.64 ; \\
4.83)\end{array}$ & \\
\hline $\begin{array}{l}\text { Gestational age } \\
\text { (weeks) }\end{array}$ & & 0.001 & & 0.044 & & 0.136 & & 0.780 & & 0.361 & & 0.575 \\
\hline$\geq 37$ & Ref & & Ref & & Ref & & Ref & & Ref & & Ref & \\
\hline$<37$ & $3.18(1.24 ; 5.12)$ & & $\begin{array}{l}1.98(0.53 ; \\
3.90)\end{array}$ & & $\begin{array}{l}-1.04(-0.33 \\
2.41)\end{array}$ & & $\begin{array}{l}-0.20 \\
(-1.62 ; 1.22)\end{array}$ & & $\begin{array}{l}0.87(-0.99 ; \\
2.73)\end{array}$ & & $\begin{array}{l}0.60(-1.49 ; \\
2.68)\end{array}$ & \\
\hline Type of delivery & & $<0.001$ & & 0.022 & & $<0.001$ & & 0.254 & & 0.015 & & 0.269 \\
\hline Vaginal & Ref & & Ref & & Ref & & Ref & & Ref & & Ref & \\
\hline Cesarean & $\begin{array}{l}-1.79(-2.68 ; \\
-0.90)\end{array}$ & & $\begin{array}{l}-1.03(-1.91 ; \\
-0.15)\end{array}$ & & $\begin{array}{l}-1.93(-2.84 \\
-1.02)\end{array}$ & & $\begin{array}{l}-0.51 \\
(-1.38 ; 0.36) \\
\end{array}$ & & $\begin{array}{l}-1.56(-2.82 \\
-0.31)\end{array}$ & & $\begin{array}{l}-0.72(-2.00 \\
0.56)\end{array}$ & \\
\hline Birth weight (g) & & 0.064 & & 0.209 & & 0.002 & & $<0.001$ & & 0.316 & & 0.038 \\
\hline$<2500$ & $1.75(0.12 ; 3.39)$ & & $\begin{array}{l}1.07(-0.65 ; \\
2.79)\end{array}$ & & $\begin{array}{l}1.75(0.12 \\
3.39)\end{array}$ & & $\begin{array}{l}3.83(2.14 \\
5.52)\end{array}$ & & $\begin{array}{l}0.85(-1.58 ; \\
3.29)\end{array}$ & & $\begin{array}{l}1.76(-1.05 ; \\
4.57)\end{array}$ & \\
\hline $2500-3499$ & $0.39(-0.48 ; 1.26)$ & & $\begin{array}{l}0.38(-0.48 ; \\
1.23)\end{array}$ & & $\begin{array}{l}0.39(-0.48 ; \\
1.26)\end{array}$ & & $\begin{array}{l}1.52(0.60 ; \\
2.44)\end{array}$ & & $\begin{array}{l}0.79(-0.64 \\
2.21)\end{array}$ & & $\begin{array}{l}1.64(0.24 \\
3.04)\end{array}$ & \\
\hline$\geq 3500$ & Ref & & Ref & & Ref & & Ref & & Ref & & Ref & \\
\hline
\end{tabular}

Table 2. Unadjusted and adjusted associations ${ }^{a}$ between predictors at birth and overall physical activity (mean acceleration $^{\mathrm{b}}$ ) in three birth cohorts in Pelotas, Brazil. ${ }^{\mathrm{a}}$ Mutually adjusted for all variables in the table, using multiple imputations for handling missing data. ${ }^{\mathrm{b}}$ In units milli-g $\left(1000 \mathrm{mg}=1 \mathrm{~g}=9.81 \mathrm{~m} / \mathrm{s}^{2}\right)$. ${ }^{\mathrm{c}}$ Cohort mean age (SD) in years: 1982 cohort: 30.2 (0.2); 1993 cohort: $18.4(0.3)$; 2004 cohort: $6.7(0.3)$. 
participants with a birthweight of $\geq 3500 \mathrm{~g}$, those with a birthweight of $<2500 \mathrm{~g}$ had 5 more minutes and those with a birthweight of 2500-3499g had nearly 2 more minutes of MVPA per day. Cesarean delivery was associated with an average of 5 minutes/day less MVPA in the 1982 cohort (Table 3).

The pattern of associations with sedentary time as the outcome was largely consistent with findings regarding physical activity outcomes, but in the opposite direction. Being male and lower family income and maternal education at birth were consistently predictive of lower sedentary time in all cohorts (Table 4). Specifically, female participants had an average of 5 more minutes of sedentary time per day than male participants in the 2004 cohort and the difference was larger in the 1993 and 1982 cohorts ( 21 and 15 minutes, respectively). The difference in sedentary time between the lowest and highest maternal education categories was around 43 minutes in both the 2004 and 1993 cohorts and 32 minutes in the 1982 cohort. The difference in sedentary time between the lowest and highest income categories was around 15 minutes in the 2004 cohort, 26 minutes in the 1993 cohort, and 29 minutes in the 1982 cohort. Mothers' BMI was positively associated with the child's sedentary time in the 1982 cohort, where those with an underweight mother had lower levels of sedentary time (around 8 minutes per day) and those with overweight or obese mother had higher levels of sedentary time (more than 9 minutes per day) than those whose mother had a normal weight. A higher birth order was associated with lower sedentary time based on unadjusted analysis in all three cohorts but was only significant in the adjusted model in the 1993 cohort, where participants with the highest birth orders (i.e., $4+$ ) had around 15 minutes less of sedentary time than firstborns. Based on unadjusted analysis, cesarean delivery was significantly associated with higher sedentary time in all three cohorts, but only significant in adjusted analysis of the 2004 cohort, where cesarean delivery was associated with 7 additional minutes/day of sedentary time.

\section{Discussion}

Findings from our study extend the current knowledge on early-life predictors of physical activity and sedentary behaviour using population-based data from a low- and middle- income country setting. Of all the prenatal and birth predictors examined, socioeconomic status at birth had the strongest and most consistent associations with physical activity and sedentary time, where children born into families with lower socioeconomic status (measured by both maternal education and family income) later became more physically active and less sedentary from childhood to adulthood. This finding echoed earlier analyses of the 1993 cohort $^{14,22}$, but contradicted the established evidence from high-income countries where people of higher socioeconomic status are more physically active $^{7}$. A systematic review based on studies primarily from high-income countries found a consistent and positive association of childhood socioeconomic status with leisure-time physical activity in adulthood and an inverse association with transport and occupational physical activity ${ }^{21}$. In high-income countries, where leisure-time physical activity accounts for a large proportion of total physical activity, higher socioeconomic status is consistently associated with higher level of overall physical activity ${ }^{7}$. In low- and middle- income countries, such as Brazil, where the majority of physical activity is not volitional in nature ${ }^{23}$, lower socioeconomic status appears consistently associated with higher levels of total and moderate-to-vigorous physical activity. The consistency of associations in all three cohorts from childhood to adulthood may be explained by limited opportunities for social mobility in Brazil $^{21}$; children born into low socioeconomic status households mostly remain disadvantaged as adults and are therefore more likely to work in labor jobs and travel actively, contributing to higher overall levels of physical activity ${ }^{22}$.

Our study found a substantial sex difference in physical activity, persistent from childhood to adulthood. Boys engage in $20+\mathrm{min} /$ day more MVPA than girls at age 6 and 18 and men did $13 \mathrm{~min} /$ day more MVPA than women at age 30. Females also had higher levels of sedentary time: although the sex difference was small at age 6 ( $5 \mathrm{~min} /$ day), it increased to $20 \mathrm{~min} /$ day at age 18 and stayed around $15 \mathrm{~min} /$ day at age 30 . In most countries around the world, women are found to be consistently less active than $\mathrm{men}^{24}$. As there is no obvious established biological explanation for the sex difference in physical activity and sedentary time, social and cultural factors may have constrained girls and women from participating in physical activity ${ }^{25}$ and it is important to target women through culturally acceptable large-scale interventions ${ }^{26}$.

Overall, birth and early life predictors of physical activity and sedentary time differ by cohort/life stage. Maternal BMI, gestational age, type of delivery and birth weight were associated with physical activity and sedentary time in some cohorts. Despite statistical significance, the effect sizes were small; and in almost all cases, the effect sizes were substantially attenuated in the adjusted models, suggesting confounding by other predictors, particularly socioeconomic status. Some evidence suggests a weak association between birth weight and physical activity later in life. A recent meta-analysis of 13 Nordic cohorts found an inverse U-shape association between birth weight and leisure-time physical activity where both very low and very high birth weights were associated with lower leisure-time physical activity ${ }^{20}$. A possible explanation is that intrauterine growth restriction compromises physical capacity and motor performance ${ }^{27}$, as extremely low birth weight has been associated with lower muscle mass and muscle strength ${ }^{28}$, developmental coordination disorder ${ }^{28}$ and poorer musculoskeletal and aerobic fitness ${ }^{27}$. Conversely, high birth weight $(>4000 \mathrm{~g}$ ) has been consistently associated with obesity, which may indirectly contribute to lower levels of physical activity and more sedentary time ${ }^{29}$. In the current study, very few participants had extremely low or high birth weight (Supplementary Table 2), which limits the opportunity to examine these aspects here. Our finding linking low birth weights to more physical activity and less sedentary time is unique, and may be explained by potential mediation by adiposity as those with lower birth weight are less likely to become obese later in life ${ }^{30}$, and therefore may consequentially be more physically active and less sedentary ${ }^{31,32}$.

Our study found a potential association between birth order and physical activity, particularly in the 1993 cohort. The pattern of association is consistent: those of higher birth order were more physically active and less sedentary in adolescence. Having siblings provides opportunities for active interactions. Thus those with the 


\begin{tabular}{|c|c|c|c|c|c|c|c|c|c|c|c|c|}
\hline \multirow[b]{2}{*}{ Variables } & \multicolumn{4}{|c|}{1982 Cohort $(\mathrm{n}=2740)^{c}$} & \multicolumn{4}{|c|}{1993 Cohort $(\mathrm{n}=3592)^{\mathrm{c}}$} & \multicolumn{4}{|c|}{2004 Cohort $(n=2603)^{c}$} \\
\hline & \begin{tabular}{|l|} 
Unadjusted $\beta$ \\
$(95 \% \mathrm{CI})$
\end{tabular} & $\mathbf{p}$ & $\begin{array}{l}\text { Adjusted } \beta \\
\text { (95\% CI) }\end{array}$ & $\mathbf{p}$ & \begin{tabular}{|l|} 
Unadjusted $\beta$ \\
$(\mathbf{9 5} \% \mathrm{CI})$
\end{tabular} & $\mathbf{p}$ & $\begin{array}{l}\text { Adjusted } \beta \\
(95 \% \mathrm{CI})\end{array}$ & $\mathbf{p}$ & \begin{tabular}{|l|} 
Unadjusted \\
$\beta(95 \% \mathrm{CI})$
\end{tabular} & $\mathbf{p}$ & $\begin{array}{l}\text { Adjusted } \beta \\
\text { (95\% CI) }\end{array}$ & $\mathbf{p}$ \\
\hline $\begin{array}{l}\text { Family income } \\
\text { (No of minimum } \\
\text { wages per month) }\end{array}$ & & $<0.001$ & & 0.005 & & $<0.001$ & & $<0.001$ & & $<0.001$ & & $<0.001$ \\
\hline$\leq 1$ & $\begin{array}{l}14.98(8.56 ; \\
21.41)\end{array}$ & & $\begin{array}{l}7.39(0.60 ; \\
14.19)\end{array}$ & & $\begin{array}{l}17.93(11.75 ; \\
25.10)\end{array}$ & & $\begin{array}{l}9.73(3.36 \\
16.10)\end{array}$ & & $\begin{array}{l}10.56(3.76 ; \\
17.37)\end{array}$ & & $\begin{array}{l}8.57(1.55 ; \\
15.58)\end{array}$ & \\
\hline $1.1-3.0$ & $\begin{array}{l}9.76(3.63 ; \\
15.90)\end{array}$ & & $\begin{array}{l}4.41(-1.93 ; \\
19.75)\end{array}$ & & $\begin{array}{l}14.26(8.62 \\
19.90)\end{array}$ & & $\begin{array}{l}7.79(2.05 \\
13.52)\end{array}$ & & $\begin{array}{l}6.51(0.07 \\
12.94)\end{array}$ & & $\begin{array}{l}5.06 \\
(-1.52 ; \\
11.63)\end{array}$ & \\
\hline $3.1-6.0$ & $\begin{array}{l}6.05(-0.46 \\
12.55)\end{array}$ & & $\begin{array}{l}2.65(-3.70 \\
9.01)\end{array}$ & & $\begin{array}{l}7.93(1.99 ; \\
13.87)\end{array}$ & & $\begin{array}{l}4.01(-1.80 ; \\
9.83)\end{array}$ & & $\begin{array}{l}2.71(-4.01 \\
9.44)\end{array}$ & & \begin{tabular}{|l|}
1.58 \\
$(-5.07$ \\
$8.22)$ \\
\end{tabular} & \\
\hline $6.1-10.0$ & $\begin{array}{l}2.80(-5.03 \\
10.64)\end{array}$ & & $\begin{array}{l}0.61(-6.85 \\
8.06)\end{array}$ & & $\begin{array}{l}3.20 \\
(-3.95 ; 10.34)\end{array}$ & & $\begin{array}{l}2.03(-4.70 \\
8.76)\end{array}$ & & $\begin{array}{l}0.76(-7.45 \\
8.97)\end{array}$ & & \begin{tabular}{|l|}
0.46 \\
$(-7.51$ \\
$8.42)$
\end{tabular} & \\
\hline$>10$ & Ref & & Ref & & Ref & & Ref & & Ref & & Ref & \\
\hline $\begin{array}{l}\text { Maternal } \\
\text { education (year } \\
\text { of schooling) }\end{array}$ & & $<0.001$ & & 0.001 & & $<0.001$ & & $<0.001$ & & 0.152 & & 0.205 \\
\hline 0 & $\begin{array}{l}13.04(7.30 \\
18.78)\end{array}$ & & $\begin{array}{l}9.14(3.03 \\
15.24)\end{array}$ & & $\begin{array}{l}23.09(13.57 ; \\
32.60)\end{array}$ & & $\begin{array}{l}16.59(7.37 \\
25.81)\end{array}$ & & $\begin{array}{l}14.80 \\
(-0.92 ; \\
30.51)\end{array}$ & & $\begin{array}{l}13.19 \\
(-1.95 ; \\
28.32)\end{array}$ & \\
\hline $1-4$ & $\begin{array}{l}\text { 10.09 (6.76; } \\
13.42)\end{array}$ & & $5.66(1.81 ; 9.51)$ & & $\begin{array}{l}12.73(8.83 ; \\
16.62)\end{array}$ & & $\begin{array}{l}6.62(2.45 \\
10.79)\end{array}$ & & $\begin{array}{l}4.24(0.14 \\
7.13)\end{array}$ & & $\begin{array}{l}0.80 \\
(-3.66 \\
5.26)\end{array}$ & \\
\hline $5-8$ & $\begin{array}{l}7.11(4.04 \\
10.18)\end{array}$ & & $3.60(0.24 ; 6.96)$ & & $\begin{array}{l}10.08(6.71 ; \\
13.45)\end{array}$ & & $5.66(2.18 ; 9.13)$ & & $\begin{array}{l}5.27(2.37 ; \\
8.17)\end{array}$ & & $\begin{array}{l}3.10(0.04 ; \\
6.16)\end{array}$ & \\
\hline $9+$ & Ref & & Ref & & Ref & & Ref & & Ref & & Ref & \\
\hline Sex & & $<0.001$ & & $<0.001$ & & $<0.001$ & & $<0.001$ & & $<0.001$ & & $<0.001$ \\
\hline Male & Ref & & Ref & & Ref & & Ref & & Ref & & Ref & \\
\hline Female & $\begin{array}{l}-13.59(-15.89 \\
-11.29)\end{array}$ & & $\begin{array}{l}-13.49(-15.73 \\
-11.26)\end{array}$ & & $\begin{array}{l}-29.07 \\
(-31.67 \\
-26.48)\end{array}$ & & $\begin{array}{l}-22.19 \\
(-31.77 \\
-26.61)\end{array}$ & & $\begin{array}{l}-21.84 \\
(-24.37 \\
-19.31)\end{array}$ & & $\begin{array}{l}-21.86 \\
(-24.42 \\
-19.29)\end{array}$ & \\
\hline $\begin{array}{l}\text { Pre-pregnancy } \\
\text { body mass index } \\
\left(\mathrm{kg} / \mathrm{m}^{2}\right)\end{array}$ & & 0.451 & & 0.347 & & 0.007 & & 0.045 & & 0.809 & & 0.909 \\
\hline$<18.5$ & $\begin{array}{l}3.60(-1.37 \\
8.58)\end{array}$ & & $\begin{array}{l}2.38(-2.15 \\
6.91)\end{array}$ & & $\begin{array}{l}9.79(4.79 \\
14.79)\end{array}$ & & $\begin{array}{l}5.81(1.17 \\
10.44)\end{array}$ & & \begin{tabular}{|l|}
-6.03 \\
$(-13.80$ \\
$1.73)$ \\
\end{tabular} & & \begin{tabular}{|l|}
-3.41 \\
$(-10.41$ \\
$3.59)$ \\
\end{tabular} & \\
\hline $18.5-24.9$ & Ref & & Ref & & Ref & & Ref & & Ref & & Ref & \\
\hline $25-29.9$ & $\begin{array}{l}-1.94(-5.38 \\
1.49)\end{array}$ & & $\begin{array}{l}-2.00(-5.13 \\
1.14)\end{array}$ & & $\begin{array}{l}-0.31 \\
3.37)\end{array}$ & & $\begin{array}{l}-0.09(-3.53 \\
3.35)\end{array}$ & & $\begin{array}{l}-0.74 \\
(-4.47 \\
2.98) \\
\end{array}$ & & \begin{tabular}{|l}
-0.63 \\
$(-4.16$ \\
$2.89)$ \\
\end{tabular} & \\
\hline$\geq 30$ & $\begin{array}{l}3.28(-2.82 \\
9.37)\end{array}$ & & $\begin{array}{l}1.80(-3.82 \\
7.42)\end{array}$ & & $\begin{array}{l}-2.12(8.64 \\
4.40)\end{array}$ & & $\begin{array}{l}-2.91(-9.01 \\
3.19)\end{array}$ & & $\begin{array}{l}-2.27 \\
(-7.23 \\
2.70)\end{array}$ & & $\begin{array}{l}-1.02 \\
(-5.95 \\
3.91)\end{array}$ & \\
\hline Birth order & & 0.009 & & 0.246 & & $<0.001$ & & 0.001 & & 0.274 & & 0.397 \\
\hline 1 & Ref & & Ref & & Ref & & Ref & & Ref & & Ref & \\
\hline 2 or 3 & $\begin{array}{l}1.60(-0.97 ; \\
4.17)\end{array}$ & & $\begin{array}{l}1.52(-0.96 ; \\
4.00)\end{array}$ & & $\begin{array}{l}1.67(-1.31 ; \\
4.66)\end{array}$ & & $\begin{array}{l}1.21(-1.60 \\
4.02)\end{array}$ & & $\begin{array}{l}-0.20 \\
(-3.09 \\
2.69)\end{array}$ & & $\begin{array}{l}0.40 \\
(-2.45 \\
3.25)\end{array}$ & \\
\hline$\geq 4$ & $4.73(1.26 ; 8.21)$ & & $\begin{array}{l}1.67(-1.85 \\
5.18)\end{array}$ & & $\begin{array}{l}11.94(7.73 ; \\
16.14)\end{array}$ & & $\begin{array}{l}7.73(3.64 \\
11.81)\end{array}$ & & $\begin{array}{l}3.13(-1.07 ; \\
7.32)\end{array}$ & & \begin{tabular}{|l|}
2.18 \\
$(-2.25$ \\
$6.61)$
\end{tabular} & \\
\hline $\begin{array}{l}\text { Gestational age } \\
\text { (weeks) }\end{array}$ & & 0.135 & & 0.240 & & 0.058 & & 0.661 & & 0.095 & & 0.464 \\
\hline$\geq 37$ & Ref & & Ref & & Ref & & Ref & & Ref & & Ref & \\
\hline$<37$ & $\begin{array}{l}4.42(-1.38 \\
10.22)\end{array}$ & & $\begin{array}{l}3.38(-2.27 \\
9.04)\end{array}$ & & $\begin{array}{l}4.35(-0.15 \\
8.85)\end{array}$ & & $\begin{array}{l}1.04(-3.62 \\
5.71)\end{array}$ & & $\begin{array}{l}3.37(-0.59 ; \\
7.32)\end{array}$ & & $\begin{array}{l}1.65 \\
(-2.77 ; \\
6.08)\end{array}$ & \\
\hline Type of delivery & & $<0.001$ & & $<0.001$ & & 0.001 & & 0.369 & & 0.241 & & 0.758 \\
\hline Vaginal & Ref & & Ref & & Ref & & Ref & & Ref & & Ref & \\
\hline Cesarean & $\begin{array}{l}-6.42(-9.08 \\
-3.77)\end{array}$ & & $\begin{array}{l}-4.62(-7.21 ; \\
-2.02)\end{array}$ & & $\begin{array}{l}-4.87(-7.85 \\
-1.89)\end{array}$ & & $\begin{array}{l}-1.32(-4.19 \\
1.56)\end{array}$ & & \begin{tabular}{|l|}
-1.60 \\
$(-4.28$ \\
$1.07)$
\end{tabular} & & $\begin{array}{l}-0.43 \\
(-3.14 \\
12.29) \\
\end{array}$ & \\
\hline Birth weight (g) & & 0.430 & & 0.634 & & 0.024 & & $<0.001$ & & 0.441 & & 0.098 \\
\hline$<2500$ & $\begin{array}{l}2.31(-2.57 \\
7.20)\end{array}$ & & $\begin{array}{l}1.22(-3.84 \\
6.28)\end{array}$ & & $\begin{array}{l}7.52(2.26 ; \\
12.78)\end{array}$ & & $\begin{array}{l}10.55 \\
16.11)\end{array}$ & & $\begin{array}{l}3.11(-2.10 \\
8.31)\end{array}$ & & \begin{tabular}{|l|}
5.05 \\
$(-0.91$ \\
$11.00)$
\end{tabular} & \\
\hline
\end{tabular}




\begin{tabular}{|c|c|c|c|c|c|c|c|c|c|c|c|c|}
\hline \multirow[b]{2}{*}{ Variables } & \multicolumn{4}{|c|}{1982 Cohort $(n=2740)^{c}$} & \multicolumn{4}{|c|}{1993 Cohort $(n=3592)^{c}$} & \multicolumn{4}{|c|}{2004 Cohort $(n=2603)^{c}$} \\
\hline & $\begin{array}{l}\text { Unadjusted } \beta \\
(95 \% \mathrm{CI})\end{array}$ & $\mathbf{p}$ & $\begin{array}{l}\text { Adjusted } \beta \\
\text { (95\% CI) }\end{array}$ & $\mathbf{p}$ & $\begin{array}{l}\text { Unadjusted } \beta \\
(95 \% \mathrm{CI})\end{array}$ & $\mathbf{p}$ & $\begin{array}{l}\text { Adjusted } \beta \\
(95 \% \text { CI })\end{array}$ & $\mathbf{p}$ & $\begin{array}{l}\text { Unadjusted } \\
\beta(95 \% \mathrm{CI})\end{array}$ & $\mathbf{p}$ & $\begin{array}{l}\text { Adjusted } \beta \\
(95 \% \text { CI })\end{array}$ & $\mathbf{p}$ \\
\hline $2500-3499$ & $\begin{array}{l}0.44(-2.16 \\
3.05)\end{array}$ & & $\begin{array}{l}0.41(-2.11 \\
2.92)\end{array}$ & & $\begin{array}{l}0.85(-2.32 \\
4.02)\end{array}$ & & $3.18(0.16 ; 6.21)$ & & $\begin{array}{l}-0.15 \\
(-3.19 \\
2.88)\end{array}$ & & $\begin{array}{l}1.72 \\
(-1.25 ; \\
4.69)\end{array}$ & \\
\hline$\geq 3500$ & Ref & & Ref & & Ref & & Ref & & Ref & & Ref & \\
\hline
\end{tabular}

Table 3. Unadjusted and adjusted associations ${ }^{\mathrm{a}}$ between predictors at birth and total moderate-to-vigorous physical activity ${ }^{\mathrm{b}}$ in three birth cohorts in Pelotas, Brazil. ${ }^{\mathrm{a}}$ Mutually adjusted for all variables in the table, using multiple imputations for handling missing data. ${ }^{\mathrm{b}}$ In units minutes/day. ${ }^{\mathrm{c}}$ Cohort mean age (SD) in years: 1982 cohort: 30.2 (0.2); 1993 cohort: 18.4 (0.3); 2004 cohort: $6.7(0.3)$.

higher birth order may benefit from having older siblings to play with ${ }^{14}$. However, another likely explanation is that birth order is a proxy for parity, which is inversely associated with socioeconomic status. Therefore, the association observed between birth order and activity outcomes may result from residual confounding by socioeconomic status. To date, very few studies have examined the association between birth order and physical activity or sedentary behaviour, and the findings have been mixed ${ }^{10,14,33}$. The observed association between birth order and activity outcomes may involve complex socioeconomic, environmental, and behavioural mechanisms. Future studies could benefit from collecting additional information, such as parity, parenting styles, and the physical activity patterns of parents and siblings.

The study used three birth cohorts including nearly all live births in each study year, with very high cohort retention rates in an low- and middle- income country setting ${ }^{34}$. Physical activity and sedentary time were measured objectively using accelerometers following standardized protocols. However, this study is subject to several limitations. First, although physical activity was measured objectively, some potential issues still apply. Accelerometer measures were collected at one time only, which does not allow for modeling long-term behavioral patterns. Physical activity level was assessed based on at least two days of valid wear time. Although including four or more days of accelerometer data may improve the reliability of the activity estimates ${ }^{35}$, for consistency with our previous paper describing the physical activity levels in the three cohorts ${ }^{36}$, we chose to include all individuals providing at least two days of data. However, it is unlikely this will affect the observed associations as the average wear time was high (i.e. 5.0 days, interquartile range 4.6-5.7 days) and did not differ by sex, socioeconomic and weight status ${ }^{36}$. Furthermore, following the previous publication ${ }^{36}$, we used the same intensity thresholds for all three cohorts. While there is currently no consensus regarding age-specific intensity thresholds from raw wrist-worn accelerometry, the patterns of descriptive physical activity data by age are in agreement with hip-worn accelerometry ${ }^{37}$, hence providing face validity to our observations. While including three cohorts with standardized data collection provides opportunities for comparison across cohorts of different ages; interpreting different patterns of associations is limited by the lack of outcome measures across cohorts at a comparable age, as it is impossible to disentangle age and cohort effects. For example, we could not determine whether the findings regarding birth order were specific to the adolescent age or the 1993 cohort. Furthermore, some predictors were measured by mothers' self-report, such as pre-pregnancy BMI, which are subject to reporting bias. Finally, while data from Brazil fill a major gap in the literature that is currently mainly based on data from high-income countries, the external validity of the study findings is yet to be determined. Given that the confounding patterns of early life predictors in low- and middle- income countries tend to differ from those observed in high-income countries ${ }^{34}$, findings from the Pelotas cohorts should be compared with other cohorts with caution and a clear understanding of the contextual differences. Birth cohort studies, particularly those from low- and middle- income country, should continue to comprehensively examine biological, behavioural, socioeconomic, and environmental influences on physical activity and sedentary time.

In summary, we found sex and socioeconomic status at birth to be the strongest predictors of physical activity and sedentary time later in life, persisting from childhood to adulthood. Low birth weight and high birth order may be associated with more physical activity and less sedentary time in Brazil. These findings should be interpreted within the context of economic development and social transitions in low- and middle- income countries, such as declines in fertility rates and improvement in neonatal health, which may lead to increasingly inactive lifestyles. Public health actions are urgently needed to prevent further declines in physical activity and increases in sedentary time at the population level.

\section{Methods}

Complete cohort profiles describing baseline characteristics and follow-up data collection have been previously published ${ }^{14,38,39}$. Briefly, participants or their legal guardians voluntarily signed a consent letter before participating in each data collection. Prenatal and birth predictors were measured objectively by trained staff or by mother's self-report. Written informed consent was obtained from all participants or their parents/legal guardians. All study protocols were approved by the Ethics Committee of the Federal University of Pelotas Medical School (affiliated with the Brazilian Federal Medical Council) and all research procedures were conducted following the approved protocols which are in accordance with relevant guidelines and regulations.

Measures. Maternal information and child anthropometric measures were collected in a standardized manner at baseline and all subsequent follow-ups to enable cross-cohort comparisons. Birth weight was measured 


\begin{tabular}{|c|c|c|c|c|c|c|c|c|c|c|c|c|}
\hline \multirow[b]{2}{*}{ Variables } & \multicolumn{4}{|c|}{1982 Cohort $(n=2740)^{c}$} & \multicolumn{4}{|c|}{1993 Cohort $(n=3592)^{c}$} & \multicolumn{4}{|c|}{2004 Cohort $(n=2603)^{c}$} \\
\hline & $\begin{array}{l}\text { Unadjusted } \\
\beta(95 \% \mathrm{CI})\end{array}$ & p & $\begin{array}{l}\text { Adjusted } \beta \\
(95 \% \text { CI })\end{array}$ & $\mathbf{p}$ & $\begin{array}{l}\text { Unadjusted } \\
\beta(95 \% \mathrm{CI})\end{array}$ & $\mathbf{p}$ & Adjusted $\beta(95 \% \mathrm{CI})$ & $\mathbf{p}$ & $\begin{array}{l}\text { Unadjusted } \\
\beta(95 \% \mathrm{CI})\end{array}$ & $\mathbf{p}$ & $\begin{array}{l}\text { Adjusted } \beta \\
(95 \% \text { CI })\end{array}$ & p \\
\hline $\begin{array}{l}\text { Family } \\
\text { income (No } \\
\text { of minimum } \\
\text { wages per } \\
\text { month) }\end{array}$ & & $<0.001$ & & 0.009 & & $<0.001$ & & $<0.001$ & & $<0.001$ & & 0.005 \\
\hline$\leq 1$ & $\begin{array}{l}-65.09 \\
(-82.86 \\
-47.31)\end{array}$ & & $\begin{array}{l}-28.75 \\
(-48.23 \\
-9.27)\end{array}$ & & $\begin{array}{l}-54.09 \\
(-67.12 \\
-41.05)\end{array}$ & & $\begin{array}{l}-25.94(-40.16 \\
-11.72)\end{array}$ & & $\begin{array}{l}-24.49 \\
(-39.06 \\
-9.92)\end{array}$ & & $\begin{array}{l}-14.74 \\
(-30.45 \\
0.97)\end{array}$ & \\
\hline $1.1-3.0$ & $\begin{array}{l}-56.48 \\
(-73.43 \\
-39.52)\end{array}$ & & $\begin{array}{l}-28.47 \\
(-46.63 \\
-10.31)\end{array}$ & & $\begin{array}{l}-47.06 \\
(-58.97 \\
-35.15)\end{array}$ & & $\begin{array}{l}-25.63(-38.43 \\
-12.83)\end{array}$ & & $\begin{array}{l}-17.39 \\
(-31.17 \\
-3.62)\end{array}$ & & $\begin{array}{l}-9.33 \\
(-24.05 \\
5.39)\end{array}$ & \\
\hline $3.1-6.0$ & $\begin{array}{l}-36.21 \\
(-54.21 \\
-18.22)\end{array}$ & & $\begin{array}{l}-19.69 \\
(-37.92 \\
-1.46)\end{array}$ & & $\begin{array}{l}-23.86 \\
(-36.40 \\
11.32)\end{array}$ & & $-9.84(-22.82 ; 3.13)$ & & $\begin{array}{l}-8.27 \\
(-22.67 \\
6.13)\end{array}$ & & $\begin{array}{l}-4.21 \\
(-19.09 \\
10.66)\end{array}$ & \\
\hline $6.1-10.0$ & $\begin{array}{l}-31.87 \\
(-53.65 \\
-10.09)\end{array}$ & & $\begin{array}{l}-23.44 \\
(-44.86 \\
-2.03)\end{array}$ & & $\begin{array}{l}-8.84 \\
(-23.96 \\
6.27)\end{array}$ & & $-2.63(-17.63 ; 12.37)$ & & $\begin{array}{l}2.03(-15.51 \\
19.59)\end{array}$ & & $\begin{array}{l}2.85 \\
(-14.98 \\
20.69)\end{array}$ & \\
\hline$>10$ & Ref & & Ref & & Ref & & Ref & & Ref & & Ref & \\
\hline $\begin{array}{l}\text { Maternal } \\
\text { education } \\
\text { (year of } \\
\text { schooling) }\end{array}$ & & $<0.001$ & & $<0.001$ & & $<0.001$ & & $<0.001$ & & $<0.001$ & & 0.008 \\
\hline 0 & $\begin{array}{l}-41.87 \\
(-57.74 \\
26.00)\end{array}$ & & $\begin{array}{l}-32.24 \\
(-49.83 \\
-14.65)\end{array}$ & & $\begin{array}{l}-60.41 \\
(-80.40 \\
-40.43)\end{array}$ & & $\begin{array}{l}-43.12(-63.75 \\
-22.50)\end{array}$ & & $\begin{array}{l}-52.79 \\
(-86.17 \\
-19.40)\end{array}$ & & $\begin{array}{l}-42.50 \\
(-76.33 \\
-8.67)\end{array}$ & \\
\hline $1-4$ & $\begin{array}{l}-47.07 \\
(-56.33 \\
-37.82)\end{array}$ & & $\begin{array}{l}-36.12 \\
(-47.20 \\
-25.03)\end{array}$ & & $\begin{array}{l}-51.82 \\
(-60.00 \\
-43.64)\end{array}$ & & $\begin{array}{l}-36.43(-45.74 \\
-27.13)\end{array}$ & & $\begin{array}{l}-18.39 \\
(-27.10 \\
-9.67)\end{array}$ & & $\begin{array}{l}-9.53 \\
(-19.53 \\
0.47)\end{array}$ & \\
\hline $5-8$ & $\begin{array}{l}-37.21 \\
(-45.74 \\
-28.68)\end{array}$ & & $\begin{array}{l}-28.72 \\
(-38.41 \\
-19.04)\end{array}$ & & $\begin{array}{l}-34.71 \\
(-41.81 \\
-27.61)\end{array}$ & & $\begin{array}{l}-23.38(-31.14 \\
-15.62)\end{array}$ & & $\begin{array}{l}-13.40 \\
(-19.56 \\
-7.23)\end{array}$ & & $\begin{array}{l}-7.49 \\
(-14.36 \\
-0.62)\end{array}$ & \\
\hline $9+$ & Ref & & Ref & & Ref & & Ref & & Ref & & Ref & \\
\hline Sex & & $<0.001$ & & $<0.001$ & & $<0.001$ & & $<0.001$ & & 0.100 & $<0.001$ & 0.007 \\
\hline Male & Ref & & Ref & & Ref & & Ref & & Ref & & Ref & \\
\hline Female & $\begin{array}{l}15.21(8.71 ; \\
21.91)\end{array}$ & & $\begin{array}{l}14.96(8.52 ; \\
21.40)\end{array}$ & & $\begin{array}{l}20.29(14.42 \\
26.17)\end{array}$ & & $20.64(14.89 ; 26.40)$ & & $\begin{array}{l}4.77(-0.92 \\
10.46)\end{array}$ & & $\begin{array}{l}5.32(0.43 ; \\
11.07)\end{array}$ & \\
\hline $\begin{array}{l}\text { Pre-pregnancy } \\
\text { body mass } \\
\text { index }\left(\mathrm{kg} / \mathrm{m}^{2}\right)\end{array}$ & & 0.027 & & 0.012 & & 0.691 & & 0.655 & & 0.476 & & 0.401 \\
\hline$<18.5$ & $\begin{array}{l}-12.23 \\
(-26.06 \\
1.60)\end{array}$ & & $\begin{array}{l}-7.50 \\
(-20.57 \\
5.57)\end{array}$ & & $\begin{array}{l}-9.06 \\
(-19.79 \\
1.67)\end{array}$ & & $-2.71(-13.08 ; 7.67)$ & & $\begin{array}{l}16.92(-0.12 ; \\
33.95)\end{array}$ & & $\begin{array}{l}9.15 \\
(-6.24 \\
24.55)\end{array}$ & \\
\hline $18.5-24.9$ & Ref & & Ref & & Ref & & Ref & & Ref & & Ref & \\
\hline $25-29.9$ & $\begin{array}{l}7.97(-1.53 \\
17.46)\end{array}$ & & $\begin{array}{l}9.29(0.10 \\
18.48)\end{array}$ & & $\begin{array}{l}-0.67 \\
(-8.54 ; 7.21)\end{array}$ & & $-0.62(-8.30 ; 7.06)$ & & $\begin{array}{l}3.18(-5.00 \\
11.36)\end{array}$ & & $\begin{array}{l}0.38 \\
(-7.48 \\
8.25)\end{array}$ & \\
\hline$\geq 30$ & $\begin{array}{l}3.91(-12.88 \\
20.71)\end{array}$ & & $\begin{array}{l}9.66 \\
(-6.62 \\
25.94)\end{array}$ & & $\begin{array}{l}-0.06 \\
(-14.11 \\
13.99)\end{array}$ & & $2.80(-10.88 ; 16.48)$ & & $\begin{array}{l}-1.77 \\
(-12.67 \\
9.13)\end{array}$ & & $\begin{array}{l}-2.70 \\
(-13.57 \\
8.18)\end{array}$ & \\
\hline Birth order & & 0.028 & & 0.256 & & $<0.001$ & & 0.001 & & 0.002 & & 0.271 \\
\hline 1 & Ref & & Ref & & Ref & & Ref & & Ref & & Ref & \\
\hline 2 or 3 & $\begin{array}{l}-0.55 \\
(-7.78 ; 6.69)\end{array}$ & & $\begin{array}{l}-0.72 \\
(-7.87 \\
6.44)\end{array}$ & & $\begin{array}{l}-10.55 \\
(-16.94 \\
-4.17)\end{array}$ & & $\begin{array}{l}-7.66(-13.94 \\
-1.38)\end{array}$ & & $\begin{array}{l}-2.77 \\
(-8.93 ; 3.39)\end{array}$ & & $\begin{array}{l}-0.81 \\
(-7.19 \\
5.57)\end{array}$ & \\
\hline$\geq 4$ & $\begin{array}{l}-12.94 \\
(-22.72 \\
-3.17) \\
\end{array}$ & & $\begin{array}{l}-6.80 \\
(-16.93 \\
3.33)\end{array}$ & & $\begin{array}{l}-28.75 \\
(-37.73 \\
-19.78)\end{array}$ & & $\begin{array}{l}-15.05(-24.17 \\
-5.93)\end{array}$ & & $\begin{array}{l}-15.87 \\
(-24.82 \\
-6.92) \\
\end{array}$ & & $\begin{array}{l}-6.67 \\
(-16.60 \\
3.26)\end{array}$ & \\
\hline $\begin{array}{l}\text { Gestational } \\
\text { age (weeks) }\end{array}$ & & 0.006 & & 0.070 & & 0.316 & & 0.739 & & 0.612 & & 0.948 \\
\hline$\geq 37$ & Ref & & Ref & & Ref & & Ref & & Ref & & Ref & \\
\hline$<37$ & $\begin{array}{l}-22.25 \\
(-38.21 \\
-6.29)\end{array}$ & & $\begin{array}{l}-14.83 \\
(-30.89 \\
1.22)\end{array}$ & & $\begin{array}{l}-4.90 \\
(-14.48 \\
4.68)\end{array}$ & & $1.78(-8.72 ; 12.28)$ & & $\begin{array}{l}-2.19 \\
(-10.65 \\
6.27)\end{array}$ & & $\begin{array}{l}-0.33 \\
(-10.24 \\
9.58)\end{array}$ & \\
\hline $\begin{array}{l}\text { Type of } \\
\text { delivery }\end{array}$ & & $<0.001$ & & 0.068 & & $<0.001$ & & 0.146 & & $<0.001$ & & 0.021 \\
\hline Vaginal & Ref & & Ref & & Ref & & Ref & & Ref & & Ref & \\
\hline Cesarean & $\begin{array}{l}16.61(9.14 \\
24.08)\end{array}$ & & $\begin{array}{l}6.95 \\
(-0.52 \\
14.42)\end{array}$ & & $\begin{array}{l}16.02(9.66 \\
22.38)\end{array}$ & & $4.75(-1.65 ; 11.16)$ & & $\begin{array}{l}10.56(4.86 \\
16.27)\end{array}$ & & $\begin{array}{l}7.16(1.09 \\
13.22)\end{array}$ & \\
\hline
\end{tabular}




\begin{tabular}{|c|c|c|c|c|c|c|c|c|c|c|c|c|}
\hline \multirow[b]{2}{*}{ Variables } & \multicolumn{4}{|c|}{1982 Cohort $(n=2740)^{c}$} & \multicolumn{4}{|c|}{1993 Cohort $(n=3592)^{c}$} & \multicolumn{4}{|c|}{2004 Cohort $(n=2603)^{c}$} \\
\hline & $\begin{array}{l}\text { Unadjusted } \\
\beta(95 \% \mathrm{CI})\end{array}$ & $\mathbf{p}$ & $\begin{array}{l}\text { Adjusted } \beta \\
(95 \% \mathrm{CI})\end{array}$ & $\mathbf{p}$ & $\begin{array}{l}\text { Unadjusted } \\
\beta(95 \% \mathrm{CI})\end{array}$ & $\mathbf{p}$ & Adjusted $\beta(95 \%$ CI $)$ & $\mathbf{p}$ & $\begin{array}{l}\text { Unadjusted } \\
\beta(95 \% \mathrm{CI})\end{array}$ & $\mathbf{p}$ & $\begin{array}{l}\text { Adjusted } \beta \\
(95 \% \text { CI })\end{array}$ & $\mathbf{p}$ \\
\hline $\begin{array}{l}\text { Birth weight } \\
\text { (g) }\end{array}$ & & $<0.001$ & & 0.261 & & $<0.001$ & & 0.006 & & 0.065 & & 0.080 \\
\hline$<2500$ & $\begin{array}{l}-12.18 \\
(-25.92 \\
1.57)\end{array}$ & & $\begin{array}{l}-2.61 \\
(-17.16 \\
11.94)\end{array}$ & & $\begin{array}{l}-16.03 \\
(-27.29 \\
-4.78)\end{array}$ & & $\begin{array}{l}-15.81(-28.29 \\
-3.34)\end{array}$ & & \begin{tabular}{|l|}
-8.18 \\
$(-19.28$ \\
$2.93)$
\end{tabular} & & \begin{tabular}{|l}
-8.25 \\
$(-21.60$ \\
$5.08)$
\end{tabular} & \\
\hline $2500-3499$ & $\begin{array}{l}-8.19 \\
(-15.51 ; \\
-0.87)\end{array}$ & & \begin{tabular}{|l}
-5.72 \\
$(-12.97$ \\
$1.53)$
\end{tabular} & & $\begin{array}{l}-7.94 \\
(-14.72 ; \\
-1.17)\end{array}$ & & $\begin{array}{l}-7.53(-14.29 \\
-0.77)\end{array}$ & & \begin{tabular}{|l}
-5.71 \\
$(-12.19$ \\
$0.77)$
\end{tabular} & & \begin{tabular}{|l}
-5.93 \\
$(-12.58$ \\
$0.73)$
\end{tabular} & \\
\hline$\geq 3500$ & Ref & & Ref & & Ref & & Ref & & Ref & & Ref & \\
\hline
\end{tabular}

Table 4. Unadjusted and adjusted associations ${ }^{\mathrm{a}}$ between predictors at birth and total sedentary time $\mathrm{e}^{\mathrm{b}}$ in three birth cohorts in Pelotas, Brazil. ${ }^{a}$ Mutually adjusted for all variables in the table, using multiple imputations for handling missing data. ${ }^{b}$ In units minutes/day. ${ }^{c}$ Cohort mean age (SD) in years: 1982 cohort: 30.2 (0.2); 1993 cohort: $18.4(0.3)$; 2004 cohort: $6.7(0.3)$.

1982 Cohort

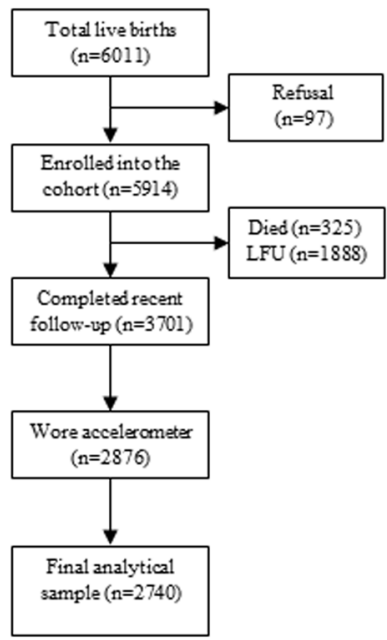

LFU $=$ lost to follow-wp
1993 Cohort

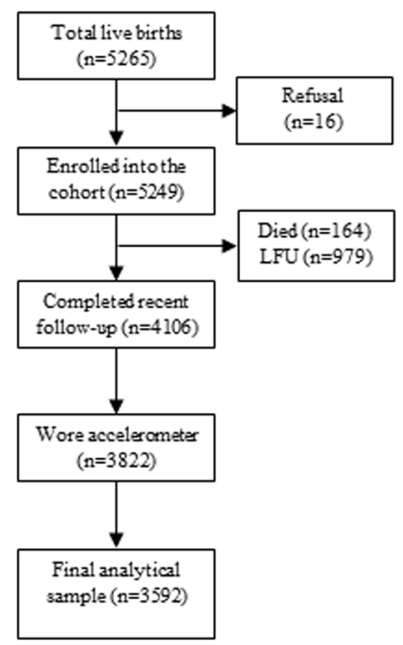

2004 Cohort

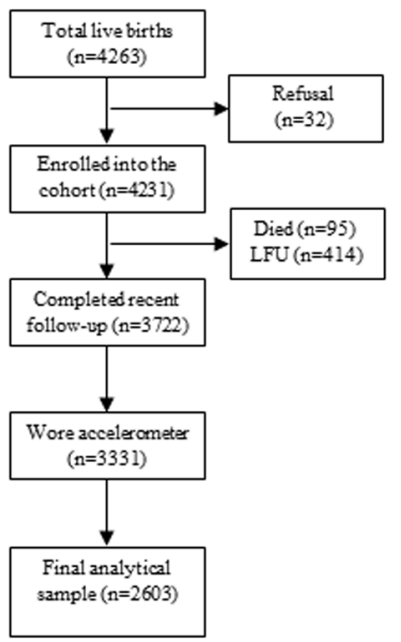

Figure 1. Participants flow.

using portable pediatric scales that were calibrated weekly by the research team (CMS and SECA) (precision $100 \mathrm{~g}$ ) and categorized in three groups as follows: $<2500 \mathrm{~g}, 2500-3499 \mathrm{~g}$, and $\geq 3500 \mathrm{~g}^{14}$. Gestational age was estimated based on the last menstrual period reported by the mothers and $<37$ weeks was defined as preterm ${ }^{40}$. Family income (categorized as $\leq 1,1.1-3.0,3.1-6.0,6.1-10.0,>10$ times the minimum wage), maternal education (years of schooling; categorized as $0,1-4,5-8$, and 9+), type of delivery (vaginal vs cesarean) and birth order $(1,2$ or 3 , and $\geq 4)$ were reported by mother at baseline. Finally, pre-pregnancy weight and height were reported by mother to calculate BMI; categorised as underweight $\left(<18.5 \mathrm{~kg} / \mathrm{m}^{2}\right)$, normal weight $\left(18.5-24.9 \mathrm{~kg} / \mathrm{m}^{2}\right)$, overweight $\left(25-29.9 \mathrm{~kg} / \mathrm{m}^{2}\right)$ and obese $\left(\geq 30 \mathrm{~kg} / \mathrm{m}^{2}\right)$.

Objectively measured physical activity and sedentary time were collected using GENEActiv (ActivInsights, Kimbolton, UK) accelerometers when participants were 30, 18, and 6 years old for the 1982, 1993, and 2004 birth cohorts, respectively. GENEActiv is a waterproof triaxial monitor, which provides raw data acceleration expressed in milli- $g$ units $\left(1000 \mathrm{~m} g=1 g=9.81 \mathrm{~m} / \mathrm{s}^{2}\right)$, directly sampled from the MEMS chip. Accelerometers were set up to collect data at $85.7 \mathrm{~Hz}$ resolution in the $+/-8 g$ range using the GENEActiv software.

Participants were invited to wear a wrist-worn accelerometer following a 24 hour-protocol including at least one weekend day. Disabled participants, those who were living outside of Pelotas, those who were unable to wear the accelerometers during work (1982 and 1993 cohorts only), and those who wore a different model of accelerometer that later was terminated unexpectedly (early stage of 2004 cohort only) were excluded from the objective physical activity and sedentary time assessment (Fig. 1). Women who were pregnant were contacted after delivery and invited to wear the accelerometer then. The analytical sample had at least 2 days of valid wear time. The average days of measurement provided by participants was $5.0(\mathrm{SD}=1.0)$ and there was no difference in wear time by sex, socioeconomic or weight status ${ }^{36}$. Further details and the complete research protocol are available elsewhere ${ }^{41}$. 
The R-package GGIR (http://cran.r-project.org) was used to analyze binary files from the GENEActiv accelerometers. A detailed signal-processing scheme and data reduction process have been described elsewhere ${ }^{42}$. In brief, three summary variables were created: overall physical activity (milli-g), time spent in (min/day) MVPA, and sedentary time (min/day). Overall physical activity is an estimate of the total volume of movement expressed by the average vector magnitude of wrist acceleration per day. MVPA was estimated as the time spent in 10-min bouts in which at least $80 \%$ of each bout was spent with accelerations equal to or higher than $100 \mathrm{mg}^{36}$. The assessment of sedentary time was restricted to an expected awake period arbitrarily defined between 7 am and $11 \mathrm{pm}$. Sedentary time was defined as non-bouted intensities lower than $50 \mathrm{~m} g^{43}$.

Data analysis. Analyses were performed using STATA software 14.2 (College Station, TX). Descriptive statistics were presented for each cohort and further compared between the baseline sample at recruitment and the analytical sample. For each outcome, we fitted general linear regression models to examine the unadjusted and adjusted associations (accounting for all other predictors in the model) between prenatal/birth predictors and a physical activity/sedentary time outcome. Results are presented as regression coefficients $(\beta)$ and 95\% CIs representing the average difference in units of each outcome by levels of exposure. Missing data (ranging from $0.01 \%$ to $14.4 \%$ ) on independent variables were handled using multiple imputation with all independent variables and the outcome variable included in each imputation model and 100 imputations $(\mathrm{m}=100)^{44}$.

Ethical approval. Federal University of Pelotas Medical School ethics committee, affiliated with the Brazilian Federal Medical Council.

Received: 17 December 2018; Accepted: 5 December 2019;

Published online: 21 January 2020

\section{References}

1. Lee, I. M. et al. Effect of physical inactivity on major non-communicable diseases worldwide: an analysis of burden of disease and life expectancy. The Lancet 380, 219-229 (2012).

2. Ding, D. et al. The economic burden of physical inactivity: a global analysis of major non-communicable diseases. Lancet 388, 1311-1324, https://doi.org/10.1016/S0140-6736(16)30383-X (2016).

3. Hallal, P. C. et al. Global physical activity levels: surveillance progress, pitfalls, and prospects. Lancet 380, 247-257, https://doi. org/10.1016/S0140-6736(12)60646-1 (2012).

4. Sallis, J. F. et al. Progress in physical activity over the Olympic quadrennium. Lancet 388, 1325-1336, https://doi.org/10.1016/S01406736(16)30581-5 (2016).

5. Sedentary Behavior Research Network. Standardized use of the terms "sedentary" and "sedentary behaviours. Applied Physiology, Nutrition and Metabolism 37, 540-542 (2012).

6. Ekelund, U. et al. Does physical activity attenuate, or even eliminate, the detrimental association of sitting time with mortality? A harmonised meta-analysis of data from more than 1 million men and women. Lancet 388, 1302-1310, https://doi.org/10.1016/ s0140-6736(16)30370-1 (2016).

7. Bauman, A. E. et al. Correlates of physical activity: why are some people physically active and others not? Lancet $\mathbf{3 8 0}, 258-271$, https://doi.org/10.1016/S0140-6736(12)60735-1 (2012).

8. O'Donoghue, G. et al. A systematic review of correlates of sedentary behaviour in adults aged 18-65 years: a socio-ecological approach. BMC Public Health 16, 163, https://doi.org/10.1186/s12889-016-2841-3 (2016).

9. Hildebrand, M., Øglund, G. P., Wells, J. C. \& Ekelund, U. Prenatal, birth and early life predictors of sedentary behavior in young people: a systematic review. Int. J. Behav. Nutr. Phys. Act. 13, 63, https://doi.org/10.1186/s12966-016-0389-3 (2016).

10. Mattocks, C. et al. Early life determinants of physical activity in 11 to 12 year olds: cohort study. BMJ 336, 26-29, https://doi. org/10.1136/bmj.39385.443565.BE (2008).

11. Barker, D. J. P. et al. Fetal nutrition and cardiovascular disease in adult life. The Lancet 341, 938-941, https://doi.org/10.1016/01406736(93)91224-A (1993).

12. Wadhwa, P. D., Buss, C., Entringer, S. \& Swanson, J. M. Developmental Origins of Health and Disease: Brief History of the Approach and Current Focus on Epigenetic Mechanisms. Semin Reprod Med 27, 358-368, https://doi.org/10.1055/s-0029-1237424 (2009).

13. Boekelheide, K. et al. Predicting later-life outcomes of early-life exposures. Environ. Health Perspect. 120, 1353-1361, https://doi. org/10.1289/ehp.1204934 (2012).

14. Hallal, P. C., Wells, J. C., Reichert, F. F., Anselmi, L. \& Victora, C. G. Early determinants of physical activity in adolescence: prospective birth cohort study. BMJ 332, https://doi.org/10.1136/bmj.38776.434560.7C (2006).

15. Biddle, S. J., Pearson, N., Ross, G. M. \& Braithwaite, R. Tracking of sedentary behaviours of young people: a systematic review. Prev. Med. 51, 345-351, https://doi.org/10.1016/j.ypmed.2010.07.018 (2010).

16. Telama, R. Tracking of physical activity from childhood to adulthood: a review. Obesity facts 2, 187-195, https://doi. org/10.1159/000222244 (2009).

17. Vickers, M. H., Breier, B. H., McCarthy, D. \& Gluckman, P. D. Sedentary behavior during postnatal life is determined by the prenatal environment and exacerbated by postnatal hypercaloric nutrition. Am J Physiol Regul Integr Comp Physiol 285, R271-273, https:// doi.org/10.1152/ajpregu.00051.2003 (2003).

18. Zhu, S., Eclarinal, J., Baker, M. S., Li, G. \& Waterland, R. A. Developmental programming of energy balance regulation: is physical activity more 'programmable' than food intake? Proc. Nutr. Soc. 75, 73-77, https://doi.org/10.1017/S0029665115004127 (2016).

19. Oglund, G. P., Hildebrand, M. \& Ekelund, U. Are Birth Weight, Early Growth and Motor Development Determinants of Physical Activity in Children and Youth? A Systematic Review and Meta-Analysis. Pediatr Exerc Sci 27, https://doi.org/10.1123/pes.20150041 (2015).

20. Andersen, L. G. et al. Birth weight in relation to leisure time physical activity in adolescence and adulthood: meta-analysis of results from 13 nordic cohorts. PLoS One 4, e8192, https://doi.org/10.1371/journal.pone.0008192 (2009).

21. Juneau, C. E., Benmarhnia, T., Poulin, A. A., Cote, S. \& Potvin, L. Socioeconomic position during childhood and physical activity during adulthood: a systematic review. Int J Public Health 60, 799-813, https://doi.org/10.1007/s00038-015-0710-y (2015).

22. Mielke, G. I. et al. Socioeconomic position and sedentary behavior in Brazilian adolescents: A life-course approach. Prev. Med. 107, 29-35, https://doi.org/10.1016/j.ypmed.2017.12.008 (2018).

23. da Silva, I. C. M. et al. Overall and Leisure-Time Physical Activity Among Brazilian Adults: National Survey Based on the Global Physical Activity Questionnaire. J. Phys. Act. Health 15, 212-218, https://doi.org/10.1123/jpah.2017-0262 (2018).

24. Guthold, R., Stevens, G. A., Riley, L. M. \& Bull, F. C. Worldwide trends in insufficient physical activity from 2001 to 2016: a pooled analysis of 358 population-based surveys with 1.9 million participants. Lancet Glob Health 6, e1077-e1086, https://doi.org/10.1016/ S2214-109X(18)30357-7 (2018). 
25. Mielke, G. I., da Silva, I. C. M., Kolbe-Alexander, T. L. \& Brown, W. J. Shifting the Physical Inactivity Curve Worldwide by Closing the Gender Gap. Sports Med. 48, 481-489, https://doi.org/10.1007/s40279-017-0754-7 (2018).

26. Ding, D. Surveillance of global physical activity: progress, evidence, and future directions. Lancet Glob Health 6, e1046-e1047, https://doi.org/10.1016/S2214-109X(18)30381-4 (2018).

27. Edwards, J. et al. Developmental Coordination Disorder in School-Aged Children Born Very Preterm and/or at Very Low Birth Weight: A Systematic Review. Journal of Developmental \& Behavioral Pediatrics 32, 678-687, https://doi.org/10.1097/ DBP.0b013e31822a396a (2011).

28. Dodds, R. et al. Birth weight and muscle strength: A systematic review and meta-analysis. The journal of nutrition, health \& aging 16, 609-615, https://doi.org/10.1007/s12603-012-0053-9 (2012).

29. Hildebrand, M. et al. Association between birth weight and objectively measured sedentary time is mediated by central adiposity: data in 10,793 youth from the International Children's Accelerometry Database. Am. J. Clin. Nutr. 101, https://doi.org/10.3945/ ajcn.114.103648 (2015).

30. Yu, Z. B. et al. Birth weight and subsequent risk of obesity: a systematic review and meta-analysis. Obes. Rev. 12, 525-542, https:// doi.org/10.1111/j.1467-789X.2011.00867.x (2011).

31. Ekelund, U. et al. Moderate to vigorous physical activity and sedentary time and cardiometabolic risk factors in children and adolescents. JAMA 307, https://doi.org/10.1001/jama.2012.156 (2012).

32. Herrmann, S. D. \& Angadi, S. S. Children's physical activity and sedentary time and cardiometabolic risk factors. Clin. J. Sport Med. 23, https://doi.org/10.1097/01.jsm.0000433154.58936.a8 (2013).

33. Giagazoglou, P. et al. The movement assessment battery in Greek preschoolers: The impact of age, gender, birth order, and physical activity on motor outcome. Res. Dev. Disabil. 32, 2577-2582, https://doi.org/10.1016/j.ridd.2011.06.020 (2011).

34. Horta, B. L. et al. Cohort Profile Update: The 1982 Pelotas (Brazil) Birth Cohort Study. Int. J. Epidemiol. 44(441), 441a-441e, https:// doi.org/10.1093/ije/dyv017 (2015).

35. Trost, S. G., Mciver, K. L. \& Pate, R. R. Conducting Accelerometer-Based Activity Assessments in Field-Based Research. Med. Sci. Sports Exerc. 37, S531-S543, https://doi.org/10.1249/01.mss.0000185657.86065.98 (2005).

36. da Silva, I. C. et al. Physical activity levels in three Brazilian birth cohorts as assessed with raw triaxial wrist accelerometry. Int. J. Epidemiol. 43, 1959-1968, https://doi.org/10.1093/ije/dyu203 (2014).

37. Hildebrand, M., VT, V. A. N. H., Hansen, B. H. \& Ekelund, U. Age group comparability of raw accelerometer output from wrist- and hip-worn monitors. Med. Sci. Sports Exerc. 46, 1816-1824, https://doi.org/10.1249/MSS.0000000000000289 (2014).

38. Santos, I. S. et al. Cohort profile: the 2004 Pelotas (Brazil) birth cohort study. Int. J. Epidemiol. 40, 1461-1468, https://doi. org/10.1093/ije/dyq130 (2011).

39. Victora, C. G. et al. Cohort profile: the 1993 Pelotas (Brazil) birth cohort study. Int. J. Epidemiol. 37, 704-709, https://doi. org/10.1093/ije/dym177 (2008).

40. World Health Organization. Preterm birth, http://www.who.int/en/news-room/fact-sheets/detail/preterm-birth (2018).

41. Knuth, A. G. et al. Methodological description of accelerometry for measuring physical activity in the 1993 and 2004 Pelotas (Brazil) birth cohorts. Cad. Saude Publica 29, 557-565 (2013).

42. van Hees, V. T. et al. Separating movement and gravity components in an acceleration signal and implications for the assessment of human daily physical activity. PLoS One 8, e61691, https://doi.org/10.1371/journal.pone.0061691 (2013).

43. Hildebrand, M., Hansen, B. H., van Hees, V. T. \& Ekelund, U. Evaluation of raw acceleration sedentary thresholds in children and adults. Scand. J. Med. Sci. Sports 27, 1814-1823, https://doi.org/10.1111/sms. 12795 (2017)

44. Sterne, J. A. et al. Multiple imputation for missing data in epidemiological and clinical research: potential and pitfalls. BMJ 338, b2393, https://doi.org/10.1136/bmj.b2393 (2009).

\section{Acknowledgements}

The authors wish to thank Joanna Sweeting (University of Sydney) for commenting on and editing the maniscript. DD is funded by a Future Leader Fellowship from National Heart Foundation of Australia. The 1982, 1993 and 2004 cohort studies were funded by: the International Development Research Center (Canada); the World Health Organization (Department of Child and Adolescent Health and Development, and Human Reproduction Programme); the Overseas Development Administration (currently Department for International Development) (UK); the European Union; the United Nations Development Fund for Women; the National Program for Centers of Excellence (PRONEX/CNPq/FAPERGS) (Brazil); the Pastorate of the Child (Brazil); the National Council for Scientific and Technological Development (CNPq), (Brazil); the Ministry of Health (Brazil), and the Wellcome Trust (WT086974MA) (UK). This work was additionally supported by the Medical Research Council [MC_ UU_12015/3] and the Research Council of Norway (249932/F20).Funders did not play any role at any stage of the study.

\section{Author contributions}

U.E. and P.C.H. conceptualised the research idea, G.I.M. prepared and managed the data, D.D. conducted the statistical analysis, D.D. and G.I.M. drafted the manuscript, all authors critically revised the manuscript and approved the final version of the manuscript.

\section{Competing interests}

The authors declare no competing interests.

\section{Additional information}

Supplementary information is available for this paper at https://doi.org/10.1038/s41598-019-57070-x.

Correspondence and requests for materials should be addressed to D.D.

Reprints and permissions information is available at www.nature.com/reprints.

Publisher's note Springer Nature remains neutral with regard to jurisdictional claims in published maps and institutional affiliations. 
(c) (i) Open Access This article is licensed under a Creative Commons Attribution 4.0 International License, which permits use, sharing, adaptation, distribution and reproduction in any medium or format, as long as you give appropriate credit to the original author(s) and the source, provide a link to the Creative Commons license, and indicate if changes were made. The images or other third party material in this article are included in the article's Creative Commons license, unless indicated otherwise in a credit line to the material. If material is not included in the article's Creative Commons license and your intended use is not permitted by statutory regulation or exceeds the permitted use, you will need to obtain permission directly from the copyright holder. To view a copy of this license, visit http://creativecommons.org/licenses/by/4.0/.

(C) The Author(s) 2020 